\title{
Intelligent remote health monitoring system
}

\section{Samik Basu, Mahasweta Ghosh and Soma Barman Mandal*}

\author{
Institute of Radio Physics and Electronics, \\ University of Calcutta, \\ 92, A.P.C. Road, Kolkata-700009, India \\ Email: samikbasu2010@gmail.com \\ Email: mahasweta94g@gmail.com \\ Email: barmanmandal@gmail.com \\ *Corresponding author
}

\begin{abstract}
With more than $45 \%$ of WHO member states failing to achieve the WHO standard of 1:1000 doctor to patient ratio; under current scenario intelligent and remote self-health monitoring becomes too vital for regular health monitoring of people. Rural people have even less doctor to patient ratio than urban people and so cost-effective intelligent vital sign monitoring system becomes even more essential. In our proposed system, we have used sensors to monitor vital health parameters like heart rate, $\mathrm{SpO}_{2}$ and body temperature and upload this information to the cloud server, using IoT platform, for remote access to the doctors. In case of non-availability of internet services, the proposed system is capable of predicting the cardiac health condition of the person independently by using machine learning algorithm. E-mail and health indicator unit alert the person at the remote-end and system-end respectively in case of emergencies.
\end{abstract}

Keywords: embedded processing; Raspberry $\mathrm{Pi} 3 \mathrm{~B}+$; health monitoring system; remote access; machine learning; support vector machine; SVM; confusion matrix; IoT; ThingSpeak cloud; green energy.

Reference to this paper should be made as follows: Basu, S., Ghosh, M. and Mandal, S.B. (2020) 'Intelligent remote health monitoring system', Int. J. Information Technology, Communications and Convergence, Vol. 3, No. 4, pp.259-275.

Biographical notes: Samik Basu received his ME in Computer Science and Engineering (specialisation in Embedded Technology) from West Bengal University of Technology (WBUT) and currently has 13+ years of experience in Semiconductor/EDA Tools industry. He served various companies in Semiconductor/EDA/Embedded domain prior to joining University of Calcutta as Project (SMDP-C2SD) Faculty where he worked as one of the key resource persons. He had setup numerous VLSI/Embedded Systems Labs in various tier-1 institute and successfully conducted workshops in several renowned academic institutes in India. His research interests are embedded system, image and video processing and machine learning.

Mahasweta Ghosh received her BTech degree from the Institute of Radio Physics and Electronics, University of Calcutta. She is currently an MTech Scholar at the Institute of Radio Physics and Electronics, University of Calcutta. She has specialisation in the field of embedded system and machine learning. 
Soma Barman Mandal received her BTech and MTech in Radio Physics and Electronics from Calcutta University, India and $\mathrm{PhD}$ (Engineering) degree from Jadavpur University, India. She is presently an Associate Professor of the Institute of Radio Physics and Electronics, University of Calcutta. Her research interest includes digital communication, digital signal processing, genomic signal processing and modelling of synthetic biological circuits, embedded system, and IoT. She is a member of the IE (India), ISTE, IEEE, and IEEE WIE Affinity Group. She has more than 75 research publications in national and international journals and conferences.

This paper is a revised and expanded version of a paper entitled 'Raspberry PI 3B+-based smart remote health monitoring system using IoT platform', presented at 2nd International Conference on Communication, Devices and Computing (ICCDC 2019), HIT, Haldia, WB, India, 14-15 March 2019.

\section{Introduction}

World Health Organization (WHO) has declared in 2018, a doctor to patient ratio of 1:1000 to be desirable by most conservative means. Until date, more than $45 \%$ of WHO member states fail to provide the standardised doctor density (WHO, 2018). India (until 2017) shows a ratio of $0.7776: 1000$ based on registered allopathy doctors alone (Kumar and Pal, 2018). There is also a problem of urban to rural doctor density, considering allopathy doctors only, i.e., there is four times more allopathy doctor density in urban regions compared to rural regions (Sudhir and Fan, 2016). In this strenuous situation, it is difficult for the patients to regularly visit a doctor for a check-up or get immediate action regarding their health. Addressing such health-related issues, we have proposed an artificially intelligent remote self-health monitoring system which will measure and display the measured vital parameters like heart rate, $\mathrm{SpO}_{2}$ and body temperature and also assess as well as predict the person's health condition.

Tseng et al. (2013) have developed a nursing home-based intelligent health monitoring system that is capable of alerting the medical staff or caregiver in case of emergency but the system is not intelligent enough to predict the health condition of the admitted patient. The overall system management is costly as it uses generally used medical sensors and also it is not suitable to monitor the health condition of a person who is not admitted to any medical centre. Yu et al. (2018) have designed a Hong Kong-based health monitoring system that caters to the needs of elderly people. In their system, they have applied artificial intelligence on the collected data but the system lacks the predicted vital parameter verification by remote doctors. It is also unable to notify medical persons during an emergency. A potential smartwatch for dementia patients has been proposed by Rajya Lakshmi et al. (2018). Their proposed system is capable of vital parameter data collection using sensors present in the smartwatch but is neither intelligent nor involves remote access to the doctor-end. Maria et al. (2017) propose a non-invasive heart-rate monitoring system using Easy Pulse PPG heart rate monitoring sensor and OLED finger-tip $\mathrm{SpO}_{2}$ sensors and analyses the measured signal. The measured data is not validated by any medical practitioner or by any published research work. Kaur et al. (2017a, 2017b) have judged the efficiency of the health monitoring system using two microprocessors: Arduino UNO and Raspberry Pi 2. In their work, they have used 
sensors like KG011 and DS18B20 to measure the vital parameters but do not involve any intelligent decision about the person's health condition. Another similar work done by Bhelkar and Shedge (2017) emphasises on the GUI and FPGA processor involved to measure the physiological parameters.

The authors in this paper have addressed a few important issues of the modern healthcare system and have attempted to mitigate such issues by using state of the art technology. The proposed intelligent remote self-health monitoring system not only measures the vital physiological parameters like heart rate, $\mathrm{SpO}_{2}$ and body temperature but also make this data available to doctors remotely using the internet of things (IoT) platform. It also uses IFTTT and Webhooks services to notify the doctor-end in case of emergency. Moreover, in case of non-availability of remote doctors or failure in internet connectivity, this system can itself intelligently predict the condition of the patients based on the measured data using machine learning (ML) algorithm. The health indicator unit of the proposed system informs the person about their health condition. A solar rechargeable power back-up unit is attached to our system which makes it eco-friendly and green. This makes the system ready-to-use in remote rural areas and during electricity failure.

The paper is organised as follows: Section 2 shows the proposed system architecture. The methodology and experimental setup of the intelligent remote health monitoring system are described in Section 3. In Section 4 the results of the training and testing of our proposed system are discussed and the paper is finally concluded in Section 5.

\section{System architecture and design}

The proposed intelligent health monitoring system can measure three vital physiological parameters - heart rate (in beats per minute), blood oxygen saturation $\mathrm{SpO}_{2}$ (in \%) and body temperature (in both ${ }^{\circ} \mathrm{C}$ and ${ }^{\circ} \mathrm{F}$ ). The system can communicate these measured data to remote doctors worldwide using IoT platform and in absence of immediate medical supervision can also predict the health condition of the person based on their age, gender and heart rate. The entire system consists of six major units - vital parameter sensing unit, embedded processing unit, local display unit, remote health monitoring unit, intelligent health predictor unit and health indicator unit. The complete block diagram representation of the proposed system is shown in Figure 1. An additional green power back-up unit is also attached to the proposed system for its uninterrupted operation under the failure of electricity.

\subsection{Vital parameter sensing unit}

The vital parameter sensing unit of the proposed system is used to measure three vital signs of the human body non-invasively. The vital signs measured by the system are heart rate (in beats per minute), capillary blood oxygen saturation $\mathrm{SpO}_{2}$ (in \%) and body temperature (in ${ }^{\circ} \mathrm{C}$ or ${ }^{\circ} \mathrm{F}$ ).

\subsubsection{Heart rate sensor and pulse oximeter}

The heart rate sensor and the pulse oximeter used in the proposed health monitoring system is a Maxim Integrated (2014) sensor Max30100. The principle of reflective 
photoplethysmography ( $r P P G$ ) is used to measure heart rate and $\mathrm{SpO}_{2}$ in $\mathrm{Max} 30100$. Allen (2007) and $\mathrm{Hu}$ et al. (2008) have established the feasibility of the use of the technique of rPPG in measuring vital parameters like heart rate and $\mathrm{SpO}_{2}$ depending on variable absorption coefficients of oxygenated and deoxygenated blood flowing through an artery or capillary for red and infrared lights. The vital measurements are taken under resting condition, from the fingertip (Longmore et al., 2019; Hertzman, 1937) where the capillaries are very close to the skin surface without much adipose tissue in between to hinder the passage of light. Hence, reflective PPG is preferred over transmission PPG (Nijboer et al., 1981). Figure 2 and Figure 3 respectively depict the mechanism of reflective PPG and the molar absorption coefficient curves for oxygenated haemoglobin $\left(\mathrm{HbO}_{2}\right)$ and reduced haemoglobin $(\mathrm{RHb})$ concentrations in blood at wavelengths $660 \mathrm{~nm}$ and $880 \mathrm{~nm}$ (Maxim Integrated, 2019).

Figure 1 The complete block diagram representation of the proposed intelligent remote health monitoring system (see online version for colours)

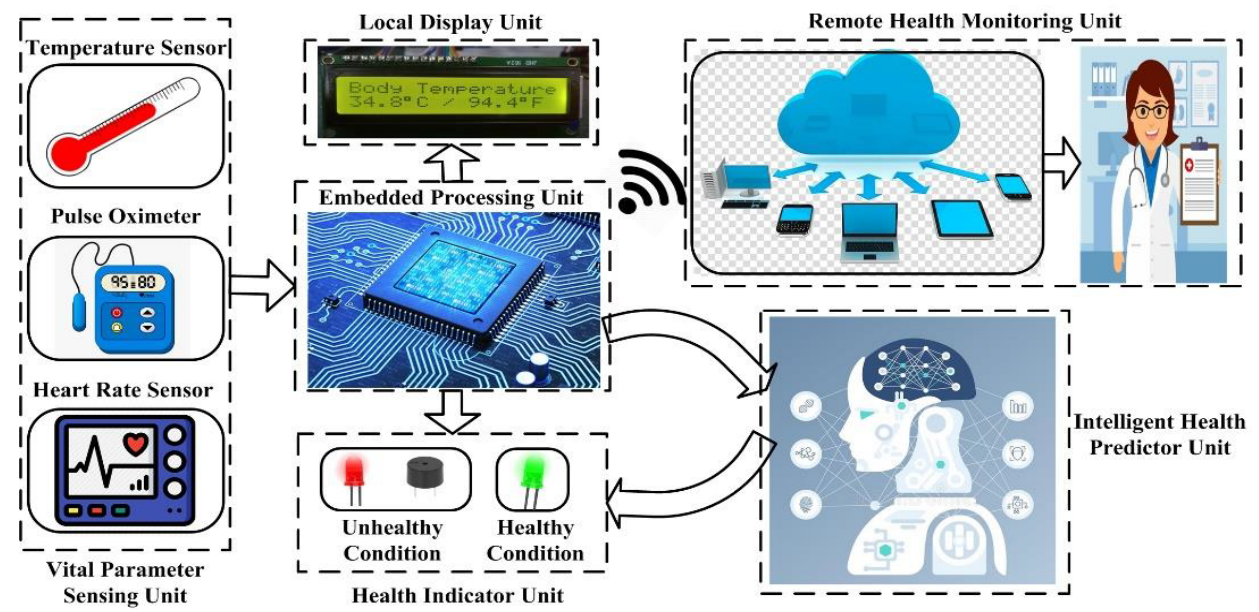

Figure 2 The technique of rPPG used in heart rate and $\mathrm{SpO}_{2}$ measurement (see online version for colours)

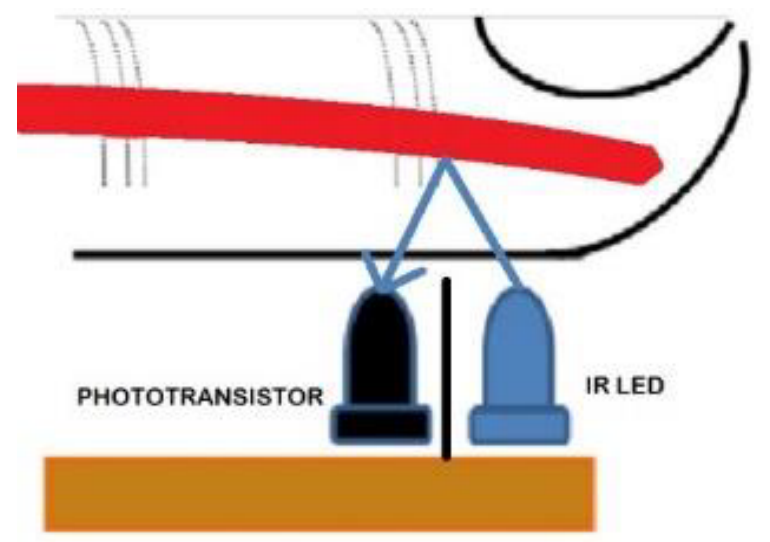


Figure 3 The molar absorption coefficient curve for $\mathrm{HbO}_{2}$ and $\mathrm{RHb}$ concentration (see online version for colours)

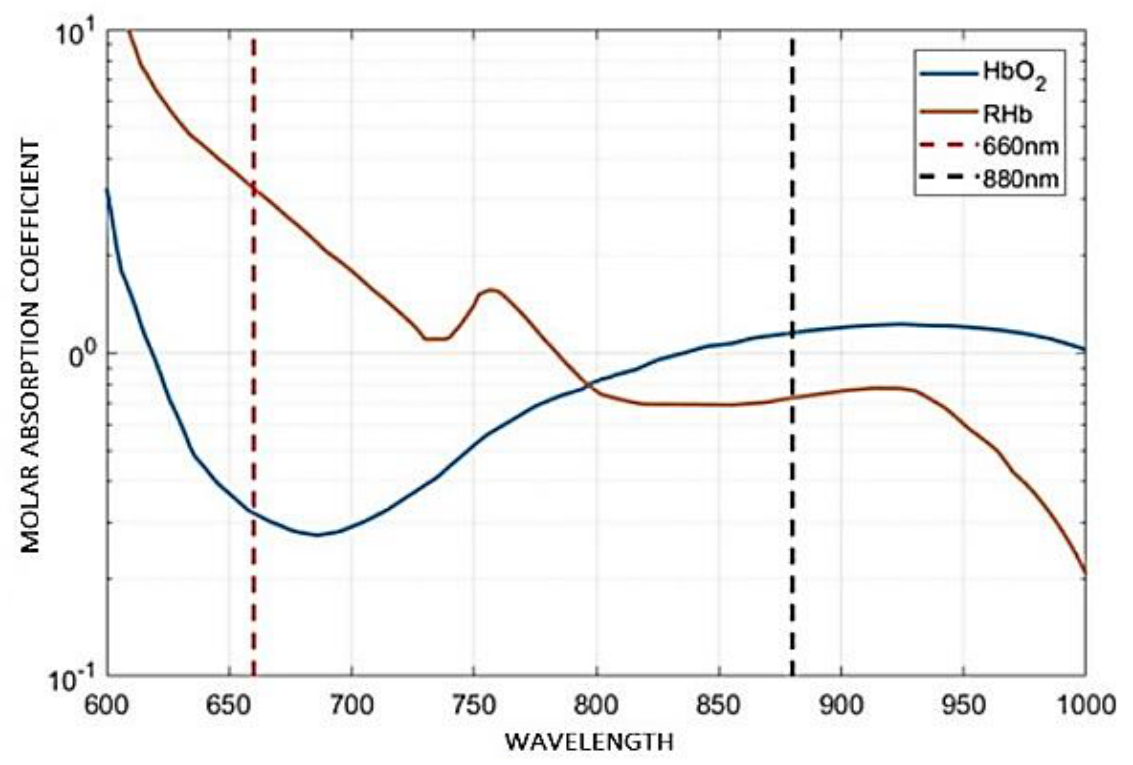

Source: Maxim Integrated (2019)

\subsubsection{Temperature sensor}

DS18B20 is used as the digital temperature sensor in our proposed system (Maxim Integrated, 2015). The probe-like arrangement allows it to measure core body temperature from any desired location like arm-pit, oral cavity, ear cavity, etc.

\subsection{Embedded processing unit}

The embedded processing unit of the intelligent remote health monitoring system comprises of both hardware and software processing tools to process the data from the sensors, store it, upload it to the cloud server and to train the intelligent health predictor unit. The hardware involved is the Raspberry $\mathrm{Pi} 3 \mathrm{~B}+$ minicomputer (Raspberry $\mathrm{Pi}$ Foundation, n.d.) and the Python 3 (Python, n.d.) is used as the software tool for coding our entire system operation.

\subsection{Local display unit}

The local display unit of our system is a $16 \times 2$ liquid crystal display (LCD) driven by a Hitachi HD44780 LCD controller. It helps the person at home to view the vital parameters measured by the sensors in a readable and understandable format. 


\subsection{Remote health monitoring unit}

The IoT platform is used to establish the communication to the doctor at the remote-end via the cloud server. In our proposed system, we have used the ThingSpeak cloud server for uploading and data storage purposes (ThingSpeak, n.d.). These vital parameter data can be observed and downloaded by the doctor at the remote-end. It can be viewed from the web-browser by the persons who have the login ID and password only. In case of any emergency, the doctor gets notified by an e-mail via the Webhooks service of IFTTT (IFTTT, n.d.).

\subsection{Intelligent health predictor unit}

The intelligent health predictor unit is trained to predict the condition of a person's heart as 'good', 'average' or 'risky' based on their heart rate, gender and age. Support vector machine (SVM) algorithm of ML has been used to train and test the system (Müller and Guido, 2016). The training set consists of the real-time data collected by using our system and the classifier is designed based on the cardiac conditions classified by the American Heart Association (AHA) as shown in Figure 4 and Figure 5 (AHA, 2015). In case of non-availability of a doctor's advice based on the measured vital parameters at any instant, this unit gives the prediction regarding the person's cardiac health.

Figure 4 The heart rate classifier design based on AHA analysis (for men)

\begin{tabular}{r|c|c|c|c|c|c|c|} 
& \multicolumn{7}{c|}{ MEN } \\
\cline { 2 - 8 } & Age & $\mathbf{1 8 - 2 5}$ & $\mathbf{2 6 - 3 5}$ & $\mathbf{3 6 - 4 5}$ & $\mathbf{4 6 - 5 5}$ & $\mathbf{5 6 - 6 5}$ & $\mathbf{6 5 +}$ \\
\cline { 2 - 8 } GOOD & Athlete & $49-55$ & $49-54$ & $50-56$ & $50-57$ & $51-56$ & $50-55$ \\
\cline { 2 - 8 } & Excellent & $56-61$ & $55-61$ & $57-62$ & $58-63$ & $57-61$ & $56-61$ \\
\cline { 2 - 8 } & Good & $62-65$ & $62-65$ & $63-66$ & $64-67$ & $62-67$ & $62-65$ \\
\cline { 2 - 8 } AVERAGE & Above Average & $66-69$ & $66-70$ & $67-70$ & $68-71$ & $68-71$ & $66-69$ \\
\cline { 2 - 8 } & Average & $70-73$ & $71-74$ & $71-75$ & $72-76$ & $72-75$ & $70-73$ \\
\cline { 2 - 8 } RISKY & Below Average & $74-81$ & $75-81$ & $76-82$ & $77-83$ & $76-81$ & $74-79$ \\
\cline { 2 - 8 } & Poor & $82+$ & $82+$ & $83+$ & $84+$ & $82+$ & $80+$ \\
\hline
\end{tabular}

Figure 5 The heart rate classifier design based on AHA analysis (for women)

\begin{tabular}{|c|c|c|c|c|c|c|c|}
\hline & \multicolumn{7}{|c|}{ WOMEN } \\
\hline & Age & $18-25$ & $26-35$ & $36-45$ & $46-55$ & $56-65$ & $65+$ \\
\hline \multirow{3}{*}{ GOOD } & Athlete & $54-60$ & 54-59 & $54-59$ & $54-60$ & $54-59$ & $54-59$ \\
\hline & Excellent & $61-65$ & $60-64$ & $60-64$ & $61-65$ & $60-64$ & $60-64$ \\
\hline & Good & $66-69$ & 65-68 & $65-69$ & $66-69$ & $65-68$ & $65-68$ \\
\hline \multirow{2}{*}{ AVERAGE } & Above Average & $70-73$ & 69-72 & $70-73$ & $70-73$ & $69-73$ & $69-72$ \\
\hline & Average & $74-78$ & $73-76$ & $74-78$ & $74-77$ & $74-77$ & $73-76$ \\
\hline \multirow{2}{*}{ RISKY } & Below Average & 79-84 & 77-82 & $79-84$ & $78-83$ & $78-83$ & $77-84$ \\
\hline & Poor & $85+$ & $83+$ & $85+$ & $84+$ & $84+$ & $84+$ \\
\hline
\end{tabular}

\subsection{Health indicator unit}

The health indicator unit contains a red LED and piezo buzzer combination and a single green LED. Whenever a person uses our proposed system to measure the vital parameters from the comforts of his/her home, the health indicator unit glows and buzzes 
appropriately. Hence, the person can get an idea about his/her overall health condition, even without any medical knowledge.

\subsection{Power back-up unit}

The system has been made eco-friendly, green and more suitable for use in remote rural areas by using solar power to drive the system. Thus, the system works uninterruptedly in the absence of electricity. Figure 6 shows the circuit designed to recharge a 10,000 mAh power bank (which supplies an output current of $2.5 \mathrm{~A}$ ) by harnessing solar power with a $12 \mathrm{~V}$ solar panel. The recharged power bank can be used to run the proposed system during a power cut or in remote locations where electricity is not yet available. The experimental arrangement during the recharging of the power bank is shown in Figure 7.

Figure 6 The circuit diagram for recharging the power bank of the power back-up unit (see online version for colours)

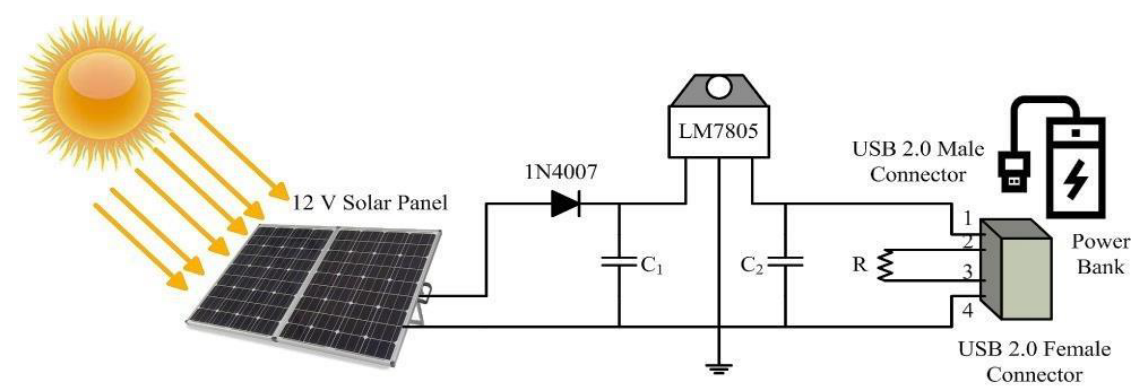

Figure 7 The experimental arrangement of the power back-up unit of the proposed system (see online version for colours)

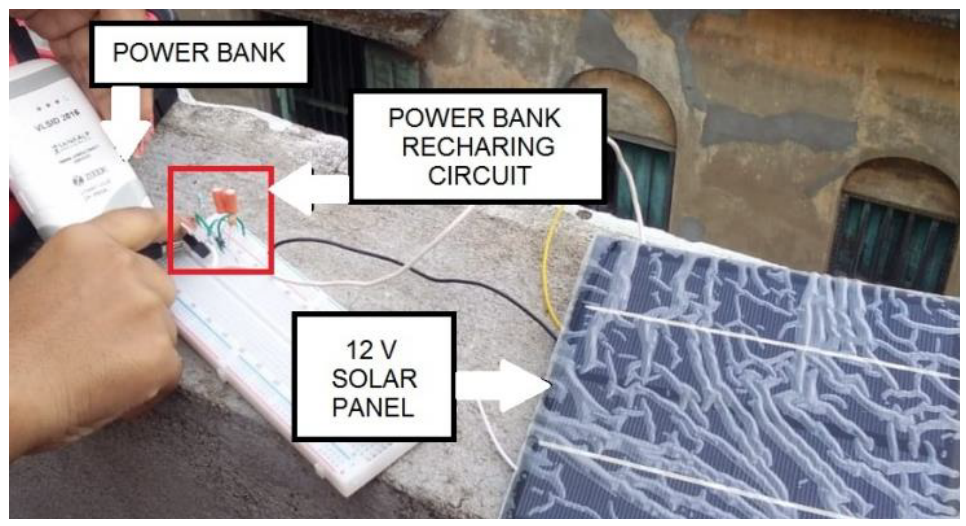

\section{Methodology}

The experimental arrangement for the proposed system is shown in Figure 8 and its complete working principle is given in Figure 9. 
Figure 8 The experimental setup of the proposed system (see online version for colours)

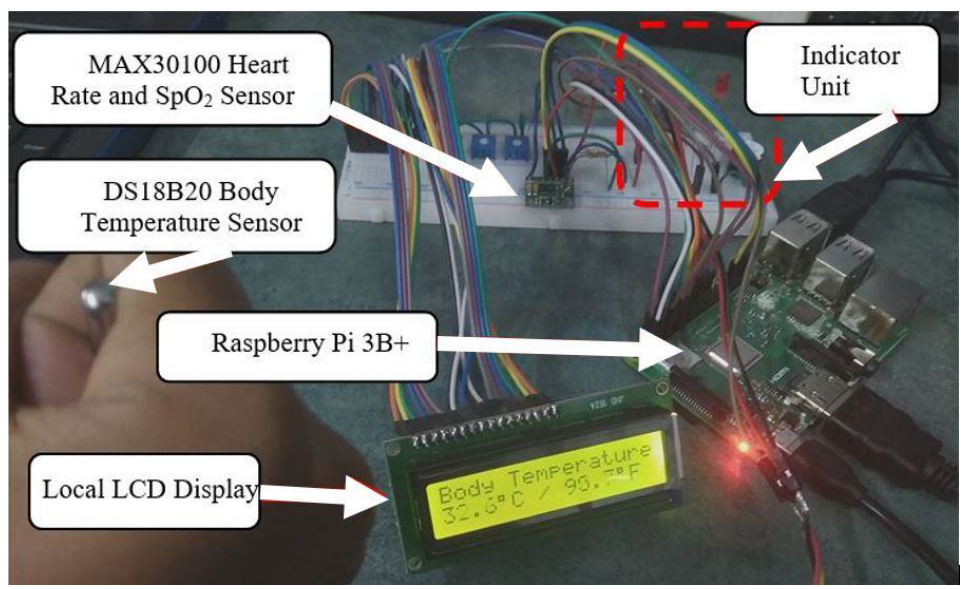

Figure 9 Flowchart of the entire intelligent health monitoring system

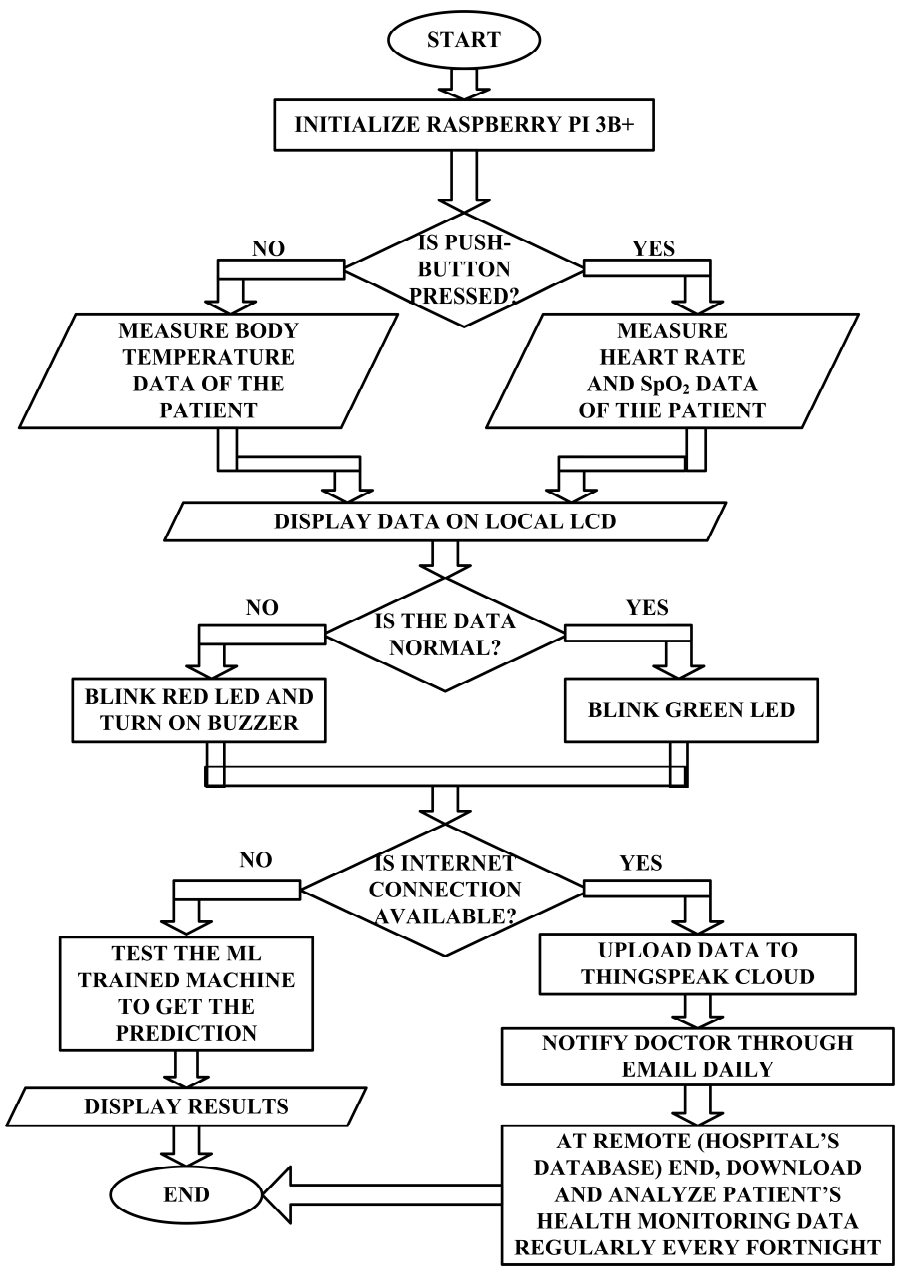




\subsection{Overall system algorithm}

The principle of operation of the intelligent remote health monitoring system is described in the following steps:

1 The system power is turned on.

2 Select the vital parameter to be measured.

a Press the push-button to measure heart rate and $\mathrm{SpO}_{2}$.

b Release the push-button to measure the body temperature.

3 Display the measured vital parameter(s) on the local LCD.

4 If the measured data is within the defined range, a green LED blinks and if not, red LED blinks and buzzer buzzes.

5 When internet connectivity is available, the measured data is uploaded to the cloud server. This data can be accessed by the doctor at the remote-end immediately or downloaded at a later time. In case of emergency, the doctor receives an e-mail stating the same.

6 However, in case of non-availability of internet services, the machine as per its training, predicts the person's cardiac condition according to the measured data and some personal information like age and gender. The predicted condition is displayed on the LCD.

\subsection{Prediction algorithm of the intelligent remote health monitoring system}

The intelligent health predictor unit employs ML algorithm to train the system. The operation principle of the unit based on ML is as follows:

1 Import datasheet.

2 Pre-process the data.

3 Normalise the data.

4 Reduce the feature dimensionality in input space to two dimensions using the principle component analysis (PCA) technique.

5 Select the kernel for the SVM algorithm. In the proposed system, the linear SVM kernel is selected.

6 Train the system.

7 Graphically represent the SVM classifier.

8 Test the trained system by predicting the labels (cardiac conditions) of the input test data.

9 Compute the normalised confusion matrix of the proposed unit.

10 Align the axes and plot the confusion matrix. 


\subsubsection{SVM prediction classifier}

In the proposed intelligent health predictor unit, multi-class linear SVM kernel has been used to train and test the unit. During this multi-class analysis, one-vs-all (OVA) or one-vs-rest (ORA) approach is utilised (Roth, n.d.). As all the classes are clearly separable (shown in Figure 4 and Figure 5), the optimisation condition to implement the classifier can be written as:

$$
\begin{aligned}
& \min _{w 1, w 2, w 3}\left\{\frac{1}{2} \sum_{k=1}^{3} w_{k}^{T} w_{k}\right\} ; \quad \text { subject to } w_{y_{i}}^{T} x-w_{k}^{T} x \geq 1 \\
& \forall\left(x_{i}, y_{i}\right) \in D \text { and } k=1,2,3
\end{aligned}
$$

The entire multi-class SVM algorithm is described in the following steps:

1 The given dataset $D$ is classified as $\left\{\left\langle x_{i}, y_{i}\right\rangle\right\}$, where $x_{i}$ denotes the input features and $y_{i}$ the output labels/markers.

2 As it is a multi-class classifier (here, $m=3$ ), so it is broken into $\frac{m(m-1)}{2}$, i.e., three binary classifiers/tasks.

3 For each of the $k^{\text {th }}$ class, i.e., the class whose output label is $y_{k}$, the classifier assigns positive values to $D$ elements having label $y_{k}$ and assigns negative values otherwise.

4 Train the ' $m$ ' binary classifiers each having weights of $w_{1}, w_{2}$ and $w_{3}$ respectively, using linear SVM equation [equation (1)] for the current OVA case.

5 Prediction is done by checking the score of the current $D$ element. The score for the true label is higher than any other label by a minimum value of 1 .

\subsubsection{PCA dimensionality reduction algorithm}

The SVM classifier uses a line to separate the two classes in a two-dimension problem but uses a hyperplane in more than 2D cases (Müller and Guido, 2016). So, we have implemented the PCA method to reduce the dimensionality of our three-feature dataset to two dimensions (Jolliffe, 2007; Dubey, 2018). The algorithm followed in PCA is shown below.

1 Take the $(D-1)$ dataset, i.e., the entire dataset ignoring the output labels. In our case $D$ is four-dimensional and on ignoring the output label $\left(y_{i}\right)$, we now have a $(4-1)$ $=$ three-dimensional dataset. So, the present dataset can be written in matrix notation as:

$$
\boldsymbol{A}=\left[\begin{array}{lll}
\boldsymbol{X}_{1} & \boldsymbol{X}_{2} & \boldsymbol{X}_{3}
\end{array}\right]
$$

where $\boldsymbol{X}_{n}$ denote the column vectors each corresponding to the three-feature dimensions $x_{i}$ of the $(D-1)$ dataset.

2 Compute the mean of every dimension of the whole $(D-1)$ dataset. Thus, the required mean matrix becomes:

$$
\bar{A}=\left[\begin{array}{lll}
\overline{X_{1}} & \overline{X_{2}} & \overline{X_{3}}
\end{array}\right]
$$


3 Compute the covariance matrix of the $(D-1)$ dataset using the formula:

$$
\operatorname{cov}(p, q)=\frac{1}{(N-1)} \sum_{i=1}^{N}\left(p_{i}-\bar{p}\right)\left(q_{i}-\bar{q}\right)
$$

Here, the covariance matrix $\mathbf{B}$ computed from $\mathbf{A}$ has a dimension of $3 \times 3$.

4 Evaluate the eigenvalues and eigenvectors of the covariance matrix $\mathbf{B}$. The eigenvalues are evaluated solving the characteristic equation:

$$
\operatorname{det}(\boldsymbol{B}-\lambda \boldsymbol{I})=0
$$

where $\mathbf{I}$ is the identity matrix. Using the eigenvalues $\lambda$, compute the eigenvectors.

5 Sort the eigenvalues in descending order.

6 Take $k$ eigenvectors corresponding to the $k$ highest eigenvalues. Use these $k$ eigenvectors to form a matrix $\mathbf{w}$ of dimension $((D-1) \times k)$. In our case, $\mathbf{w}$ has a dimension of $(3 \times 2)$.

7 Transform the samples into a new subspace given by the equation:

$$
z=\boldsymbol{w}^{T} x
$$

$\mathbf{w}^{T}$ is the transform of $\mathbf{w}$ and has a dimension of $(k \times(D-1))$, i.e., $(2 \times 3)$.

Thus, we get a rwo-dimensional feature space for SVM optimisation.

\section{Results and analysis}

The vital parameter data as recorded by the proposed system is uploaded immediately to the ThingSpeak cloud server whenever the internet connection is available and the uploaded data for a single person is shown in Figure 10, Figure 11 and Figure 12.

Figure 10 The body temperature data of a person as measured by the DS18B20 sensor of the proposed system (see online version for colours)

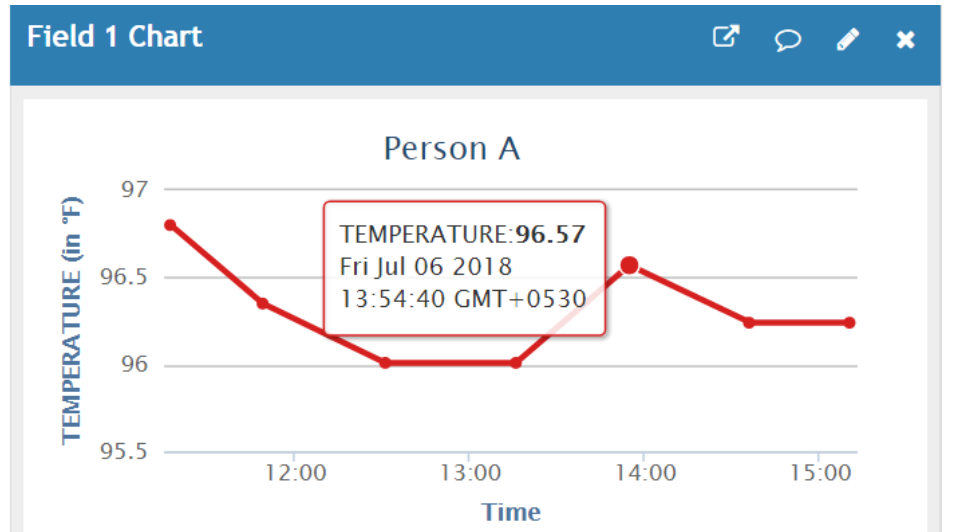


Figure 11 The heart rate data of a person as measured by theMAX30100 sensor of the proposed system (see online version for colours)
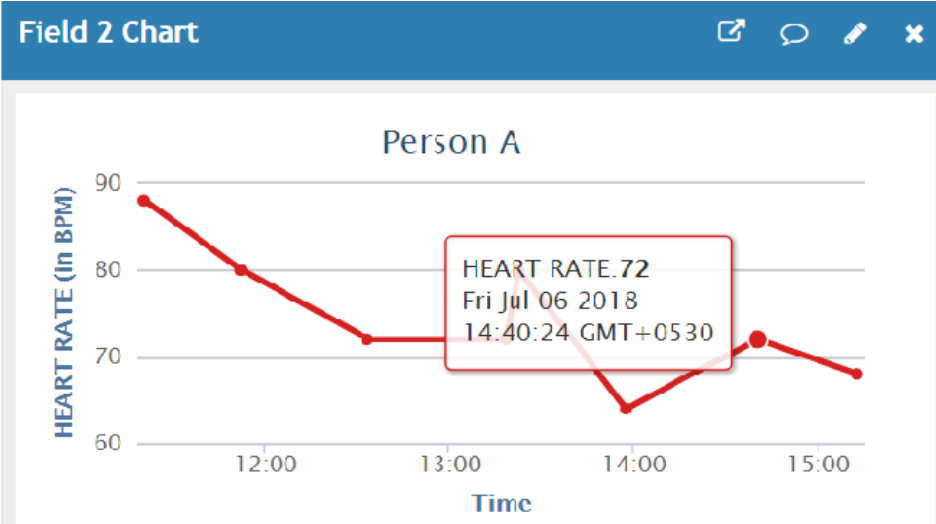

Thingspeak.com

Figure 12 The $\mathrm{SpO}_{2}$ data of a person as measured by theMAX30100 sensor of the proposed system (see online version for colours)
Field 3 Chart
$\square 0, x$

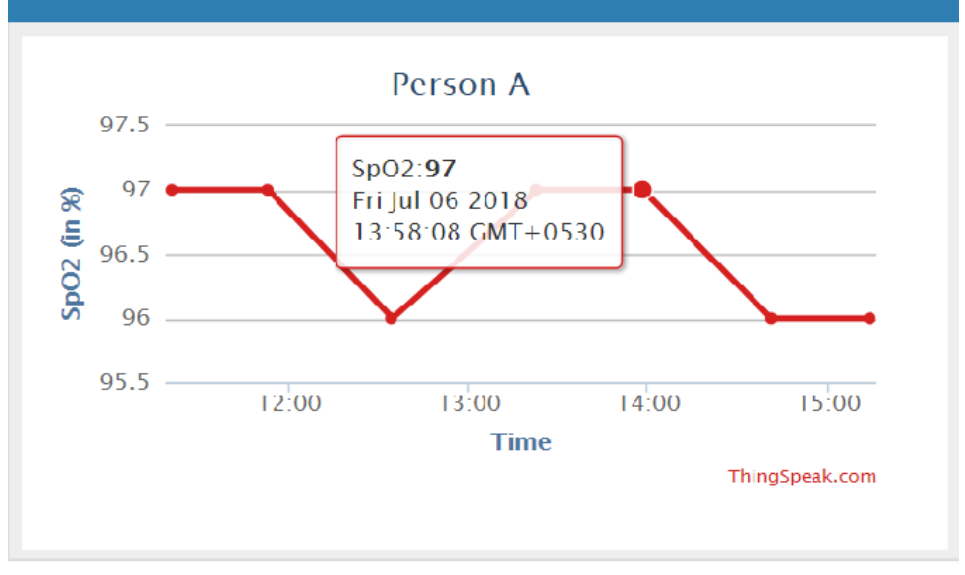

Table 1 shows a small portion of our training dataset along with the classifier labels for each dataset.

The SVM analysis plot for the trained system is given in Figure 13. When the proposed system is tested, the normalised confusion matrix for the test results is shown in Figure 14. The validation of the system using the test data shows that the system gives $100 \%$ correct prediction for 'GOOD' and 'RISKY' vital data. However, the true 'AVERAGE' data is predicted correctly in $71 \%$ cases but predicted incorrectly as 'GOOD' condition in $18 \%$ cases and as 'RISKY' in the leftover $12 \%$ cases. 
Table 1 A few training data along with their labels used for developing the intelligent health predictor unit

\begin{tabular}{|c|c|c|c|}
\hline Age (in years) & Gender & Heart rate (in bpm) & Target class \\
\hline 24 & FEMALE & 93 & RISKY \\
\hline 24 & FEMALE & 84 & AVERAGE \\
\hline 24 & MALE & 60 & GOOD \\
\hline 24 & MALE & 64 & GOOD \\
\hline 24 & MALE & 72 & AVERAGE \\
\hline 24 & MALE & 88 & RISKY \\
\hline 4 & FEMALE & 92 & RISKY \\
\hline 24 & FEMALE & 84 & AVERAGE \\
\hline 24 & MALE & 100 & RISKY \\
\hline 24 & MALE & 68 & GOOD \\
\hline 24 & MALE & 68 & GOOD \\
\hline 27 & FEMALE & 70 & AVERAGE \\
\hline 29 & MALE & 104 & RISKY \\
\hline 26 & MALE & 68 & GOOD \\
\hline 26 & MALE & 75 & AVERAGE \\
\hline 48 & MALE & 84 & RISKY \\
\hline 26 & MALE & 80 & AVERAGE \\
\hline 45 & FEMALE & 71 & AVERAGE \\
\hline 24 & FEMALE & 66 & GOOD \\
\hline 24 & MALE & 60 & GOOD \\
\hline 22 & MALE & 100 & RISKY \\
\hline 23 & FEMALE & 80 & AVERAGE \\
\hline 24 & FEMALE & 88 & RISKY \\
\hline 24 & FEMALE & 100 & RISKY \\
\hline 24 & FEMALE & 72 & AVERAGE \\
\hline 26 & MALE & 76 & AVERAGE \\
\hline 26 & MALE & 78 & AVERAGE \\
\hline 27 & MALE & 88 & RISKY \\
\hline 21 & MALE & 68 & GOOD \\
\hline 38 & MALE & 80 & AVERAGE \\
\hline 33 & FEMALE & 88 & RISKY \\
\hline 32 & FEMALE & 106 & RISKY \\
\hline 33 & MALE & 100 & RISKY \\
\hline 26 & MALE & 80 & AVERAGE \\
\hline 29 & FEMALE & 84 & RISKY \\
\hline 30 & MALE & 84 & RISKY \\
\hline 30 & MALE & 84 & RISKY \\
\hline
\end{tabular}


Table 1 A few training data along with their labels used for developing the intelligent health predictor unit (continued)

\begin{tabular}{lccc}
\hline Age (in years) & Gender & Heart rate (in bpm) & Target class \\
\hline 41 & MALE & 104 & RISKY \\
20 & MALE & 68 & GOOD \\
52 & MALE & 100 & RISKY \\
48 & MALE & 76 & AVERAGE \\
24 & FEMALE & 64 & GOOD \\
24 & FEMALE & 109 & RISKY \\
24 & FEMALE & 102 & RISKY \\
24 & MALE & 84 & RISKY \\
24 & MALE & 84 & RISKY \\
41 & MALE & 64 & GOOD \\
24 & FEMALE & 76 & AVERAGE \\
20 & MALE & 92 & RISKY \\
21 & MALE & 74 & AVERAGE \\
24 & FEMALE & 76 & AVERAGE \\
24 & FEMALE & 100 & RISKY \\
21 & MALE & 84 & RISKY \\
21 & MALE & 84 & RISKY \\
46 & MALE & 88 & RISKY \\
\hline
\end{tabular}

Figure 13 The SVM plot for the trained machine using heart rate data for cardiac health prediction (see online version for colours)

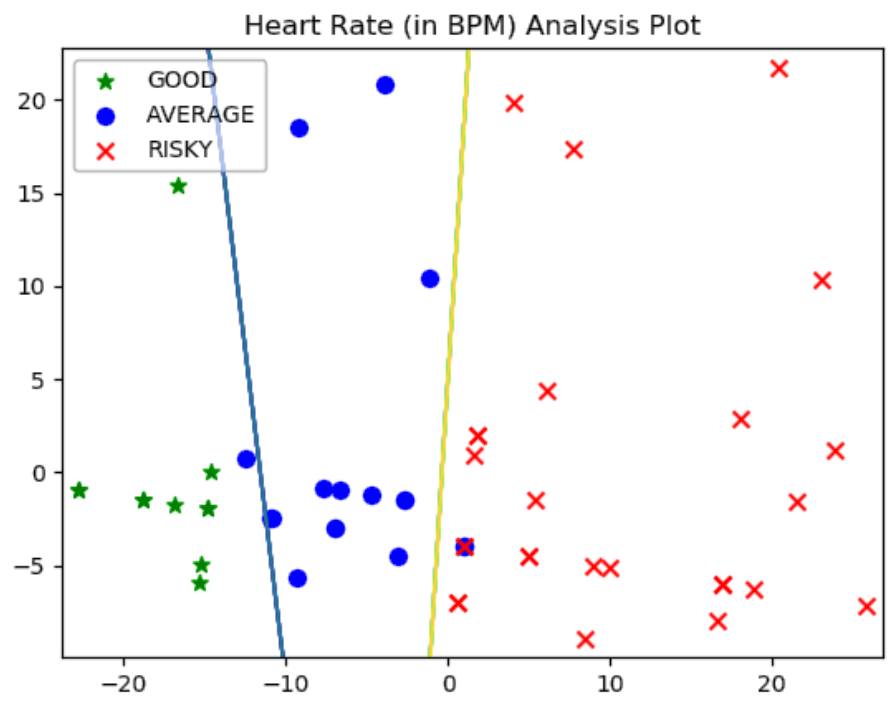


Figure 14 The normalised confusion matrix for the trained machine using heart rate data for cardiac health prediction (see online version for colours)

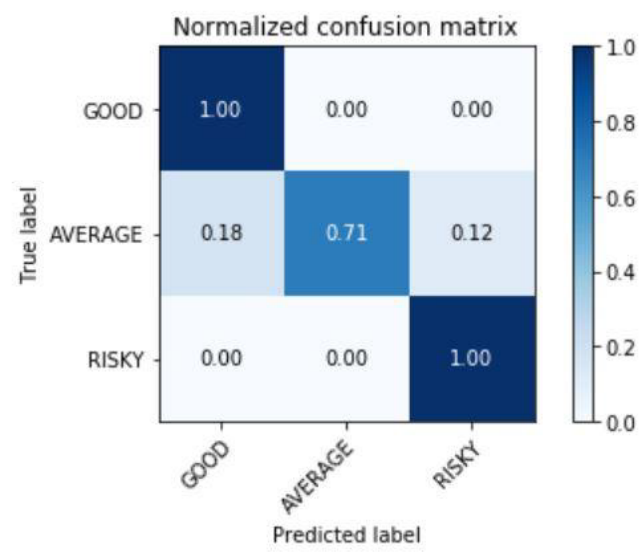

The proposed system is a complete package and is better than the surveyed literatures in the following aspects:

- It is a complete self-health monitoring system which is home-based and hence can be used to monitor a person's health at his convenience.

- Use of solar power to recharge the power back-up unit to run our system makes it green.

- The system can be used even in remote or rural areas as it is easily portable and has an additional rechargeable power back-up unit.

- $\quad$ This system is capable of distant communication using IoT platform to access physicians even from remote areas.

- It also uses IFTTT and Webhooks services to send e-mail notifications to the doctor-end in case of emergency.

- Using ML algorithm to train the proposed system for health condition prediction makes the system intelligent.

\section{Conclusions}

This paper presents an eco-friendly, low power, accurate, intelligent, remote health monitoring system which has a potential to monitor and predict the health condition of any person and also can seek the advice of a remote doctor by using state of the art IoT technology. Use of solar recharge power back-up arrangement makes our system green.

This proposed system has a scope of further augmentation by including more sensors to measure vital parameters like ECG, blood pressure, respiration rate, etc. Also, telemedicine services can be incorporated with the proposed system to make a complete home-based intelligent self-health service package. 


\section{Acknowledgements}

The authors would like to thank UGC UPE II project 'Modern Biology: Signal Processing Group', Calcutta University and SMDP-C2SD project, University of Calcutta funded by MeitY, Government of India for providing funding and technical support. The work does not have any conflict of interest.

\section{References}

Allen, J. (2007) 'Photoplethysmography and its application in clinical physiological measurement', Physiological Measurement, Vol. 28, No. 3, DOI: 10.1088/0967-3334/28/3/r01.

American Heart Association (AHA) (2015) All About Heart Rate (Pulse), 31 July [online] http://www.heart.org/HEARTORG/Conditions/HighBloodPressure/GettheFactsAboutHighBlo odPressure/All-About-Heart-Rate-Pulse_UCM_438850_Article.jsp\#.Wz811dIza00 (accessed 3 May 2018).

Bhelkar, V. and Shedge, D.K. (2017) 'Design and implementation of health monitoring device using FPGA', International Journal of Computer Applications, Vol. 158, No. 7, pp.8-11, DOI: $10.5120 /$ ijca2017912841.

Dubey, A. (2018) The Mathematics Behind Principal Component Analysis, 25 December [online] https://towardsdatascience.com/the-mathematics-behind-principal-component-analysisfff2d7f4b643 (accessed 7 November 2018).

Hertzman, A.B. (1937) 'Photoelectric plethysmography of the fingers and toes in man', Experimental Biology and Medicine, Vol. 37, No. 3, pp.529-534, DOI: 10.3181/00379727-379630.

Hu, S., Zheng, J., Chouliaras, V. and Summers, R. (2008) 'Feasibility of imaging PPG', International Conference on BioMedical Engineering and Informatics, pp.72-75, DOI: 10.1109/BMEI.2008.365.

IFTTT (n.d.) [online] https://ifttt.com/maker_webhooks (accessed 13 January 2019).

Jolliffe, I.T. (2007) Principal Component Analysis, Springer, New York.

Kaur, A. and Jasuja, A. (2017a) 'Cost-effective remote health monitoring system based on IoT using Arduino UNO', Advances in Computer Science and Information Technology (ACSIT), Vol. 4, No. 2, pp.80-84.

Kaur, A. and Jasuja, A. (2017b) 'Health monitoring based on IoT using Raspberry Pi', 2017 International Conference on Computing, Communication and Automation (ICCCA), pp.1335-1340, DOI: 10.1109/CCAA.2017.8230004.

Kumar, R. and Pal, R. (2018) 'India achieves WHO recommended doctor population ratio: a call for paradigm shift in public health discourse!', Journal of Family Medicine and Primary Care, Vol. 7, No. 5, pp.841-844, DOI: 10.4103/jfmpc.jfmpc_218_18.

Longmore, S.K., Lui, G.Y., Naik, G., Breen, P.P., Jalaludin, B. and Gargiulo, G.D. (2019) 'A comparison of reflective photoplethysmography for detection of heart rate, blood oxygen saturation, and respiration rate at various anatomical locations', Sensors, Vol. 19, No. 8, p.1874, DOI: $10.3390 / \mathrm{s} 19081874$.

Maria, A.R., Pasca, S. and Strungaru, R. (2017) 'Heart rate monitoring by using non-invasive wearable sensor', 2017 E-Health and Bioengineering Conference (EHB), DOI: 10.1109/ ehb.2017.7995492.

Maxim Integrated (2014) MAX30100 Pulse Oximeter and Heart-Rate Sensor IC for Wearable Health [online] https://datasheets.maximintegrated.com/en/ds/MAX30100.pdf (accessed 6 June 2018).

Maxim Integrated (2015) DS18B20 Programmable Resolution 1-Wire Digital Thermometer, Rev. 4 [online] https://datasheets.maximintegrated.com/en/ds/DS18B20.pdf (accessed 12 June 2018). 
Maxim Integrated (2019) Guidelines for $\mathrm{SpO}_{2}$ Measurement Using the Maxim ${ }^{\circledR}$ MAX32664 Sensor $H u b, 26$ March [online] https://www.maximintegrated.com/en/app-notes/index.mvp/id/6845 (accessed 24 December 2018).

Müller, A.C. and Guido, S. (2016) Introduction to Machine Learning with Python a Guide for Data Scientists, OReilly, Beijing.

Nijboer, J.A., Dorlas, J.C. and Mahieu, H.F. (1981) 'Photoelectric plethysmography-some fundamental aspects of the reflection and transmission methods', Clinical Physics and Physiological Measurement, Vol. 2, No. 3, pp.205-215, DOI: 10.1088/0143-0815/2/3/004.

Python (n.d.) Python 3.7.4 Documentation [online] https://docs.python.org/3 (accessed 5 January 2019).

Rajya Lakshmi, P.N.V., Venkatesan, P. and Prasad, G.R. (2018) 'Smart watch for healthcare monitoring', International Journal of Engineering Technology Science and Research, Vol. 5, No. 3, pp.1107-1111.

Raspberry Pi Foundation (n.d.) [online] https://www.raspberrypi.org/documentation (accessed 19 February 2020).

Roth, D. (n.d.) CS 546 Machine Learning in NLP. Multiclass classification: Local and Global Views [online] http://12r.cs.uiuc.edu/ danr/Teaching/CS546-16/Lectures/Lec3-multiclass.pdf.

Sudhir, A. and Fan, V. (2016) 'The health workforce in India', Human Resources for Health Observer, World Health Organization, Geneva, Switzerland.

ThingSpeak (n.d.) Understand Your Things [online] https://thingspeak.com/ (accessed 10 January 2019).

Tseng, K.C., Hsu, C-L. and Chuang, Y-H. (2013) 'Designing an intelligent health monitoring system and exploring user acceptance for the elderly', Journal of Medical Systems, Vol. 37, No. 6, DOI: 10.1007/s10916-013-9967-y.

World Health Organization (WHO) (2018) Density of Physicians (Total Number per 1000 Population, Latest Available Year), 28 December [online] https://www.who.int/gho/health workforce/physicians_density/en/ (accessed 22 April 2019).

Yu, L., Chan, W.M., Zhao, Y. and Tsui, K.L. (2018) 'Personalized health monitoring system of elderly wellness at the community level in Hong Kong', IEEE Access, Vol. 6, pp.35558-35567, DOI: 10.1109/access.2018.2848936. 